\title{
Unexplained Hyperthyroglobulinemia in Differentiated Thyroid Cancer Patients as an Indication for Radioiodine Adjuvant Therapy: A Prospective Multicenter Study
}

\author{
Lin Cheng*1 ${ }^{* 1}$, Ri Sa*1, Qiong Luo*2, Hao Fu ${ }^{1}$, Yuchen Jin ${ }^{1}$, Linglin Tang ${ }^{3}$, Yi Yang ${ }^{4}$, Chunjing Yu ${ }^{5}$, and Libo Chen ${ }^{1}$ \\ ${ }^{1}$ Department of Nuclear Medicine, Shanghai Jiao Tong University Affiliated Sixth People's Hospital, Shanghai, China; ${ }^{2}$ Department \\ of Nuclear Medicine, Tenth People's Hospital of Tongji University, Shanghai, China; ${ }^{3}$ Department of Nuclear Medicine, Ren Ji \\ Hospital, School of Medicine, Shanghai Jiao Tong University, Shanghai, China; ${ }^{4}$ Department of Nuclear Medicine, Affiliated Suzhou \\ Science and Technology Town Hospital of Nanjing Medical University, Suzhou, China; and ${ }^{5}$ Department of Nuclear Medicine, \\ Affiliated Hospital of Jiangnan University, Wuxi, China
}

The management for totally thyroidectomized differentiated thyroid cancer (TT-DTC) patients with unexplained hyperthyroglobulinemia remains indeterminate because of evidence scarcity. This multicenter study aimed at prospectively evaluating the response to radioiodine $\left({ }^{131} \mathrm{I}\right)$ adjuvant therapy (RAT) and its potential role in risk stratification and causal clarification. Methods: TT-DTC patients with stimulated serum thyroglobulin levels greater than $10 \mathrm{ng} / \mathrm{mL}$ but no structurally evident disease were consecutively enrolled in 5 tertiary-care institutions. After the administration of $5.55 \mathrm{GBq}$ of ${ }^{131}$, the risk of persistent, recurrent, or metastatic differentiated thyroid cancer (prmDTC) was compared with that before RAT. The causes of hyperthyroglobulinemia were explored-and the response to RAT assessed-6-12 mo after RAT. The change in suppressed thyroglobulin level was reported. Results: A cohort of 254 subjects with a median stimulated thyroglobulin level of $27.1 \mathrm{ng} / \mathrm{mL}$ was enrolled for the analyses. Immediately after RAT, low, intermediate, and high risk were identified in $5.9 \%, 88.6 \%$, and $5.5 \%$ patients, respectively, with no significant difference in risk stratification compared with that before RAT $(P=0.952)$. During the follow-up (median, 10.6 $\mathrm{mo}$ ), hyperthyroglobulinemia was ultimately attributed to a thyroid remnant, biochemical disease, and structural or functional disease in $17.3 \%, 54.3 \%$, and $28.4 \%$ of subjects, respectively. In addition, responses that were excellent, indeterminate, biochemically incomplete, and structurally or functionally incomplete were achieved in $18.1 \%, 27.2 \%, 36.2 \%$, and $18.5 \%$ of patients, respectively. Notably, the distribution for either cause of hyperthyroglobulinemia or response to RAT was comparable among the 3 postoperative risk groups. Suppressed thyroglobulin levels in patients who merely received RAT declined significantly over time. Conclusion: Our study demonstrated that over $90 \%$ of TT-DTC patients with unexplained hyperthyroglobulinemia are stratified as being at intermediate to high risk, and RAT using $5.55 \mathrm{GBq}$ of ${ }^{131}$ I reveals biochemical, functional, or structural disease and yields a non-structurally or -functionally incomplete response in more than $80 \%$ patients, suggesting that TT-DTC patients with unexplained hyperthyroglobulinemia are explicit candidates for RAT.

Received Feb. 20, 2020; revision accepted Apr. 9, 2020.

For correspondence or reprints contact: Libo Chen, Shanghai Jiao Tong University Affiliated Sixth People's Hospital, 600 Yishan Rd., Shanghai 200233, China.

E-mail: Ibchen@sjtu.edu.cn

${ }^{*}$ Contributed equally to this work.

Published online May 1, 2020.

COPYRIGHT (C 2021 by the Society of Nuclear Medicine and Molecular Imaging.

Key Words: differentiated thyroid cancer; thyroglobulin; thyroidectomy; radioiodine; adjuvant therapy

J Nucl Med 2021; 62:62-68

DOI: 10.2967/jnumed.120.243642

$\mathbf{W}$

th the exception of active surveillance and thermal ablation in patients with low-risk microscopic papillary thyroid cancer, the initial management of differentiated thyroid cancer relies mainly on surgery followed by radioiodine $\left({ }^{131} \mathrm{I}\right)$ remnant ablation (RRA), radioiodine adjuvant therapy (RAT) of potential residual thyroid cancer, or radioiodine treatment (RT) of known disease, under thyroid-stimulating hormone (TSH) suppression (1-3).

Before ${ }^{131} \mathrm{I}$ administration, the postoperative serum thyroglobulin level measured at either the stimulated $\left(\mathrm{Tg}_{\text {off }}\right)$ or the suppressed $\left(\mathrm{Tg}_{\text {on }}\right) \mathrm{TSH}$ level is of great value in both disease monitoring and management decision making, since hyperthyroglobulinemia a few weeks after total thyroidectomy is highly associated with persistent, recurrent, or metastatic differentiated thyroid cancer (prmDTC) and survival (4-8). The 2015 American Thyroid Association (ATA) guidelines pointed out that patients with postoperative $\mathrm{Tg}_{\text {off }}$ levels of more than $10 \mathrm{ng} / \mathrm{mL}$ will likely need additional evaluations and possibly even complementary therapies (1). Unfortunately, although it has been over $10 \mathrm{y}$ since the term thyroglobulinemia was initially raised by the well-acknowledged 2009 ATA guidelines (9), few dedicated data exist for totally thyroidectomized differentiated thyroid cancer (TT-DTC) patients with unexplained hyperthyroglobulinemia, partially because of inclusion of patients with structural disease in previous studies $(7,10)$. Consequently, the relationship between hyperthyroglobulinemia and the risk of carrying prmDTC, the primary cause of unexplained hyperthyroglobulinemia, and the response to ${ }^{131}$ I administration in such patients remains largely unknown.

Aiming at facilitating initial staging and follow-up, improving disease-specific survival and progression-free survival, and decreasing recurrence, RAT should be considered or routinely recommended in intermediate- or high-risk patients but not in low-risk patients, with regard to guidelines issued by the ATA (1). However, the implications of serum thyroglobulin level in risk stratification have not been fully clarified, and the attempt to define the indications for RAT has been retarded, since a precise thyroglobulin cutoff has not been 
TABLE 1

Baseline Characteristics of TT-DTC Patients with Postoperative Unexplained Hyperthyroglobulinemia $(n=254)$

\begin{tabular}{|c|c|}
\hline Characteristics & Data \\
\hline \multicolumn{2}{|l|}{ Age $(n)$} \\
\hline$<55$ y & $220(86.6 \%)$ \\
\hline$\geq 55 \mathrm{y}$ & $34(13.4 \%)$ \\
\hline \multicolumn{2}{|l|}{$\operatorname{Sex}(n)$} \\
\hline Female & $158(62.2 \%)$ \\
\hline Male & $96(37.8 \%)$ \\
\hline \multicolumn{2}{|l|}{ Pathologic subtype (n) } \\
\hline Papillary & 248 (97.6\%) \\
\hline Follicular & $6(2.4 \%)$ \\
\hline \multicolumn{2}{|l|}{ pT stage $(n)$} \\
\hline $\mathrm{T} 1$ & $154(60.6 \%)$ \\
\hline $\mathrm{T} 2$ & 44 (17.3\%) \\
\hline T3 & $29(11.4 \%)$ \\
\hline $\mathrm{T} 4$ & 15 (5.9\%) \\
\hline Tx & $12(4.7 \%)$ \\
\hline \multicolumn{2}{|l|}{ pN stage $(n)$} \\
\hline No & $13(5.1 \%)$ \\
\hline $\mathrm{N} 1$ & $223(87.8 \%)$ \\
\hline $\mathrm{Nx}$ & $18(7.1 \%)$ \\
\hline \multicolumn{2}{|l|}{ Stage $(n)$} \\
\hline I & 230 (90.5\%) \\
\hline II & $22(8.7 \%)$ \\
\hline III & $2(0.8 \%)$ \\
\hline $\begin{array}{l}\text { Median postoperative } \\
\operatorname{Tg}_{\text {on }}(\mathrm{ng} / \mathrm{mL})\end{array}$ & 3.7 (IQR, 1.3-9.3) \\
\hline $\begin{array}{l}\text { Median postoperative } \\
\operatorname{Tg}_{\text {off }}(\mathrm{ng} / \mathrm{mL})\end{array}$ & 27.1 (IQR, 16.1-47.1) \\
\hline $\begin{array}{l}\text { Median TSH at } 4 \text { wk after } \\
\text { THW (mIU/L) }\end{array}$ & 100 (range, 49.5-100) \\
\hline $\begin{array}{l}\text { Mean } 24-\mathrm{h} \text { radioactive iodine } \\
\text { uptake } \pm \mathrm{SD}(\%)\end{array}$ & $4.7 \pm 3.1$ \\
\hline $\begin{array}{l}\text { Visualization of thyroid bed on } \\
\text { post-RAT scan }(n)\end{array}$ & 249 (98.0\%) \\
\hline
\end{tabular}

$\mathrm{IQR}=$ interquartile range; THW = thyroid hormone withdrawal.

firmly established for distinguishing intermediate- from low- or highrisk patients or for distinguishing RAT from RRA or RT. Hence, we conducted this prospective multicenter study to assess the response to RAT and its potential role in risk stratification and causal clarification in a dedicated cohort of TT-DTC patients with unexplained hyperthyroglobulinemia regardless of postoperative risk stratification, to establish a potential indication for RAT.

\section{MATERIALS AND METHODS}

\section{Patients}

TT-DTC patients with hyperthyroglobulinemia, whose $\mathrm{Tg}_{\text {off }}$ levels at more than 4 wk after total thyroidectomy and just before RAT were more than $10 \mathrm{ng} / \mathrm{mL}$, were consecutively recruited from June 2009 in 5 tertiary-care institutions. Patients with a prmDTC lesion identified by neck ultrasonography (1), chest CT, ${ }^{99 \mathrm{~m}} \mathrm{Tc}$-methylene diphosphonate whole-body scanning, MRI, or ${ }^{18} \mathrm{~F}-\mathrm{FDG} \mathrm{PET} / \mathrm{CT}$ were excluded. Consequently, a $\mathrm{Tg}_{\text {off }}$ level of over $10 \mathrm{ng} / \mathrm{mL}$ in TT-DTC patients without structurally evident prmDTC was defined as unexplained hyperthyroglobulinemia and comprehensively analyzed.

Just before RAT, patients with radioiodine uptake greater than $15 \%$ were excluded to ensure the success of RRA as previously described by our group (11). Serum TSH, thyroglobulin, and antithyroglobulin antibody ( $\mathrm{TgAb}$ ) levels were measured by electrochemiluminescence immunoassay on a Cobas analyzer (Roche Diagnostics Gmbh). The upper detection limit of the TSH assay was 100 $\mathrm{mIU} / \mathrm{L}$, and TSH levels higher than that were counted as $100 \mathrm{mIU} / \mathrm{L}$. Similarly, thyroglobulin and $\mathrm{TgAb}$ levels lower than their thresholds were counted as $0.04 \mathrm{ng} / \mathrm{mL}$ and $10 \mathrm{IU} / \mathrm{mL}$, respectively. Neck ultrasonography and chest $\mathrm{CT}$ were conducted on all patients before RAT.

For RAT, an activity of $5.55 \mathrm{GBq}$ of ${ }^{131} \mathrm{I}$ was orally administered. Three days later, a planar whole-body scan was performed with SPECT/CT if needed (12). RT was performed 6 mo after RAT in patients if ${ }^{131} \mathrm{I}$-avid prmDTC lesions were identified by post-RAT scanning; conversely, patients without overt structural or functional disease were regularly followed up for more than $1 \mathrm{y}$ under TSH suppression. $\mathrm{Tg}_{\text {on }}$ levels were tested and neck ultrasonography performed at each follow-up visit. The follow-up visit, and possible modulation of levothyroxine dosage, were done at 1,4 , and 12 mo after RAT except as otherwise indicated, then every 6-12 mo. The assessment of therapeutic response to RAT and the primary cause of hyperthyroglobulinemia were revealed 6-12 mo after RAT.

The institutional review boards approved this study, and all subjects gave written informed consent.

\section{Risk Stratification}

According to the 2009 ATA guidelines, the 3-tiered system, including a low, intermediate, and high risk of prmDTC, was used with minor revisions (9). Low-risk patients were characterized by intrathyroidal differentiated thyroid cancer with no evidence of extrathyroidal extension, vascular invasion, aggressive histology, or metastases. Intermediate-risk patients were featured by either microscopic extrathyroidal extension, cervical lymph node metastases, ${ }^{131} \mathrm{I}$-avid disease in the neck outside the thyroid bed, vascular invasion, or aggressive tumor histology. High-risk patients were distinguished by either gross extrathyroidal extension, incomplete tumor resection, or distant metastases.

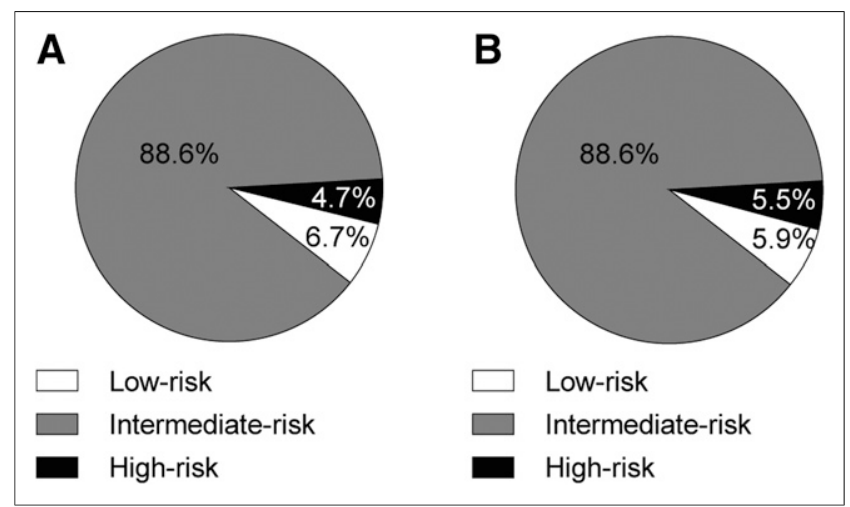

FIGURE 1. Risk stratifications in patients with unexplained hyperthyroglobulinemia before (A) and just after (B) RAT $(n=254)$. 


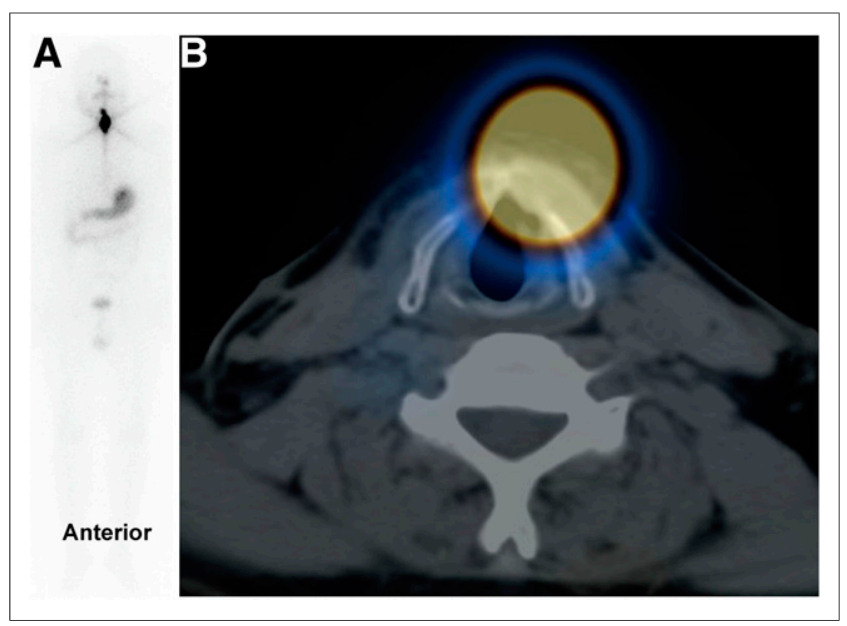

FIGURE 2. Post-RAT whole-body scan (A) and SPECT/CT image (B) of papillary thyroid carcinoma patient with unexplained hyperthyroglobulinemia causally attributed to thyroid remnant.

\section{Causal Categorization}

The primary causes of postoperative unexplained hyperthyroglobulinemia were based on the findings of post-RAT scanning, consecutive serum thyroglobulin tests, medical imaging, ultrasonographyguided fine-needle aspiration in combination with pathologic examination, and correlation with clinical follow-up within 6-12 mo after RAT

Determination of a thyroid remnant was based on visible uptake in the normal thyroid bed or ectopia on post-RAT scanning, absence of prmDTC, and a $\mathrm{Tg}_{\text {on }}$ level of less than $0.2 \mathrm{ng} / \mathrm{mL}$. Biochemical disease was affirmed by the absence of structural or functional evidence of prmDTC but a $\mathrm{Tg}_{\text {on }}$ level of at least $0.2 \mathrm{ng} / \mathrm{mL}$. Structural or functional disease was defined as any evidence of prmDTC lesions identified by post-RAT scanning or other imaging examinations during surveillance.

\section{Response to RAT}

According to the 2015 ATA guidelines, a response evaluation scheme with 4 categories was adopted with minor modifications 6-12 mo after RAT (1). The definitions of excellent response, indeterminate response, and biochemically incomplete response remained. A structurally or functionally incomplete response (S/FIR) endorsed positive anatomic or metabolic imaging evidence of disease with any thyroglobulin level. Indeterminate response, biochemically incomplete response, and S/FIR were collectively assigned as a nonexcellent response, whereas an excellent response, an indeterminate response, and a biochemically incomplete response were grouped as non-S/FIR.

\section{Statistical Analyses}

All analyses were conducted using SPSS (version 20; IBM) software. Continuous data are presented as means with SDs or medians with interquartile ranges for normal and abnormal distributions, respectively. Categoric variables are displayed as number with percentage. Categoric variables were compared using the $\chi^{2}$ test or Fisher exact test. Variance was analyzed for continuous data with a normal distribution. The predictive value of $\mathrm{Tg}_{\text {off }}$ for the primary cause of hyperthyroglobulinemia was evaluated by univariate receiver-operating-characteristic analysis in combination with the DeLong test for comparing the area under the curve. The Wilcoxon signed-rank test for thyroglobulin level was performed at multiple time points. Two-tailed probabilities were reported, and a $P$ value of less than 0.05 was considered statistically significant.

\section{RESULTS}

\section{Baseline Characteristics}

From June 2009 through August 2018, 469 TT-DTC patients with a $\mathrm{Tg}_{\text {off }}$ level of more than $10 \mathrm{ng} / \mathrm{mL}$ were enrolled. After the exclusion of 215 patients ( 8 patients with $\mathrm{TgAb}>100 \mathrm{IU} / \mathrm{mL}, 55$ patients with radioiodine uptake $>15 \%, 114$ patients with suspected cervical locoregional disease, and 38 patients with possible distant metastases), 54.2\% (254/469) of patients deemed as having unexplained hyperthyroglobulinemia were eligible for further analyses.

The baseline characteristics of the 254 subjects are summarized in Table 1. The mean age at diagnosis was $38.3 \pm 11.8 \mathrm{y}$, with a male-to-female ratio of 1:1.65. In total, 252 (99.2\%) patients were classified as stage I or II. None of the enrolled patients had undergone RRA before RAT. The mean interval between surgery and RAT was $4.2 \pm 3.3$ mo. The postoperative median $\mathrm{Tg}_{\text {on }}$ level was $3.7 \mathrm{ng} / \mathrm{mL}$, while thyroglobulin level increased up to $27.1 \mathrm{ng} / \mathrm{mL}$ just before RAT, when the median TSH level was $100 \mathrm{mIU} / \mathrm{mL}$ at 4 wk after thyroid hormone withdrawal.

\section{Risk Stratification}

In the postoperative risk stratification, only 17 (6.7\%) of the 254 eligible patients were categorized into the low-risk group. In total, $225(88.6 \%)$ and $12(4.7 \%)$ patients were classified as having an intermediate and high risk, respectively, of harboring prmDTC. Once post-RAT scan findings were obtained, $11.8 \%(2 / 17)$ of postoperative low-risk patients switched to the intermediate-risk group for the identification of nodule disease, replacing another 2 intermediate-risk patients who were transferred to the high-risk group because of distant metastasis. Thus, the number of patients with intermediate risk remained at 225 . No significant difference in the risk distribution between the 2 time points (before and after RAT) was achieved $(P=0.952)$ (Fig. 1).

\section{Causal Categorization}

According to the findings in a median follow-up of 10.6 mo (range, 7.3-13.8 mo), the unexplained postoperative hyperthyroglobulinemia

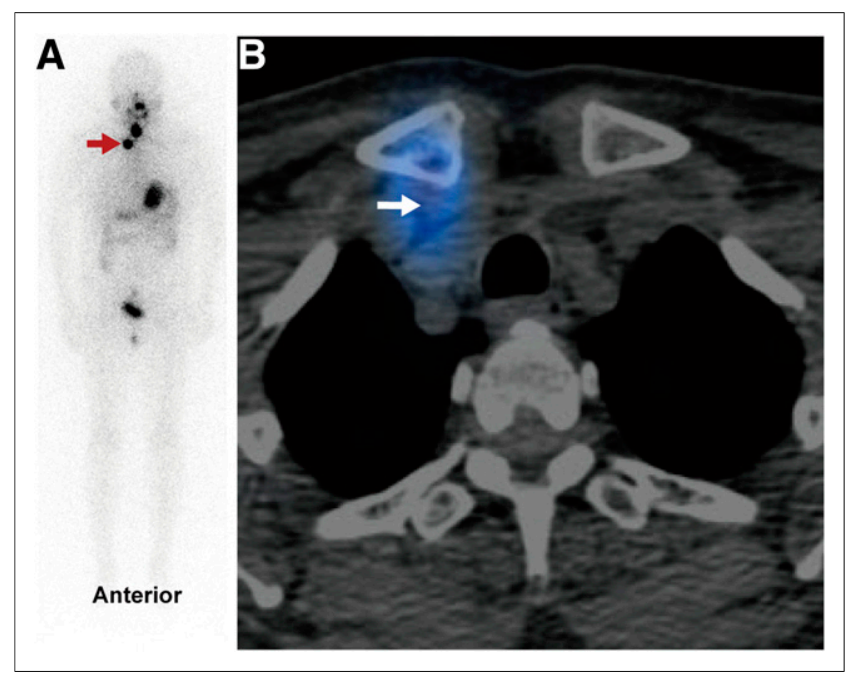

FIGURE 3. Post-RAT whole-body scan (A) and SPECT/CT image (B) of papillary thyroid carcinoma patient with unexplained hyperthyroglobulinemia causally attributed to metastatic lymph node (arrows) unable to be diagnosed by ultrasonography and CT before RAT. 


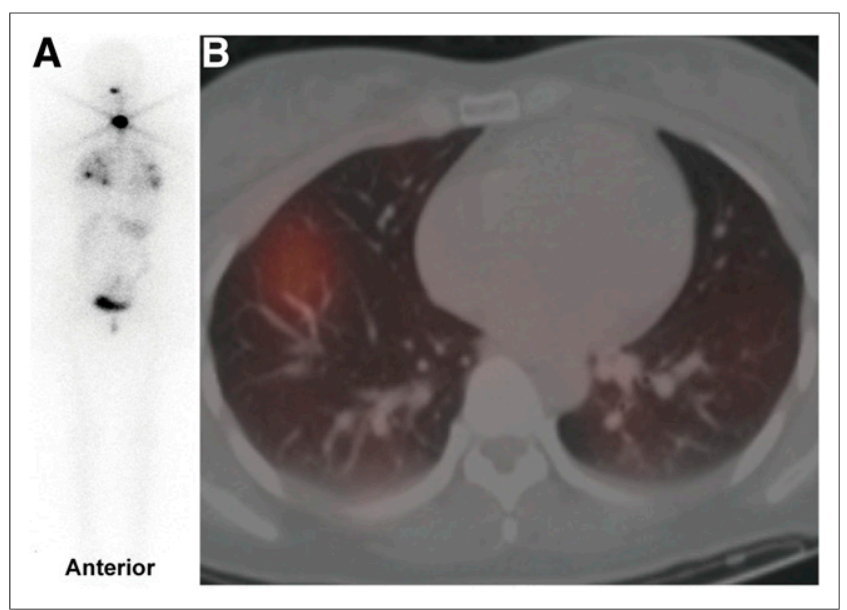

FIGURE 4. Post-RAT whole-body scan (A) and SPECT/CT image (B) of follicular thyroid carcinoma patient with unexplained hyperthyroglobulinemia causally attributed to lung metastases unable to be identified by CT before RAT.

could ultimately be attributed to a thyroid remnant in $17.3 \%$ (Fig. 2), biochemical disease in $54.3 \%$, and structural or functional disease in 28.4\% (Figs. 3 and 4) of the 254 patients (Fig. 5).

The causal classification of the postoperative risk of prmDTC is shown in Table 2. Again, no significant difference in causal proportion was achieved among the 3 risk groups $(P=0.526)$. Moreover, the proportion of either biochemical or structural or functional disease in the intermediate- to high-risk group was comparable with that in the low-risk group $(P=0.465)$.

Furthermore, the comparisons of factors potentially associated with the cause of unexplained hyperthyroglobulinemia in TT-DTC patients are shown in Table 3 . The median $\mathrm{Tg}_{\text {off }}$ level significantly differed among the 3 causal groups $(P=0.000)$. The $\mathrm{Tg}_{\text {off }}$ cutoff for distinguishing a thyroid remnant from biochemical, structural, or functional disease was $19.5 \mathrm{ng} / \mathrm{mL}$, with an area under the curve of 0.714 , a sensitivity of $68.2 \%$, and a specificity of $70.0 \%(P<0.0001)$. In addition, a $\mathrm{Tg}_{\text {off }}$ level of $27.9 \mathrm{ng} / \mathrm{mL}$ was obtained as the cutoff for isolating structural or functional disease from a thyroid remnant and biochemical disease, with an area under the curve of 0.630 , a sensitivity of $68.2 \%$, and a specificity of $58.8 \%(P=0.0009)$. However, no significant difference in radioiodine uptake, TSH, or TgAb level was achieved among the 3 causal groups, with a comparable proportion of patients having a TSH of more than $100 \mathrm{mIU} / \mathrm{mL}$ or a $\mathrm{TgAb}$ of less than $10 \mathrm{IU} / \mathrm{mL}$.

Of the 72 patients causally attributed to structural or functional disease, $38(52.8 \%)$ received RT because of the ${ }^{131} \mathrm{I}$ avidity of prmDTCs as demonstrated by post-RAT scanning, in which 1, 2, and 3 courses of RT were given in $33(86.8 \%), 3(7.9 \%)$, and 2 $(5.3 \%)$ patients, respectively. Thirty-four patients were identified with prmDTC during the 6- to 12-mo follow-up, in which 3 underwent cervical lymph node resection and the remaining $31(43.1 \%)$ were under active surveillance with TSH suppression.

Additionally, 2 patients with anatomically negative but functionally positive disease were identified. One with pulmonary metastases achieved negative results on ${ }^{131}$ I whole-body scanning after the second therapeutic dose, and one with iliac metastasis, who received $\mathrm{RT}$ followed by bone lesion resection, obtained a disease-free status in the subsequent $1-y$ follow-up.

\section{Response to RAT}

Overall, 46 (18.1\%), 69 (27.2\%), 92 (36.2\%), and $47(18.5 \%)$ of the 254 eligible patients achieved excellent response, indeterminate response, biochemical incomplete response, and S/FIR, respectively (Fig. 5).

Regarding postoperative risk stratification, an excellent response was achieved in $35.3 \%$ (6/17) of low-risk patients, $16.4 \%(37 / 225)$ of intermediate-risk patients, and $25.0 \%(3 / 12)$ of high-risk patients $(P=0.119)$. Besides, S/FIR was obtained in $11.8 \%(2 / 17)$ of lowrisk patients, $19.6 \%(44 / 225)$ of intermediate-risk patients, and $8.3 \%(1 / 12)$ of high-risk patients $(P=0.632)$.

After exclusion of 41 patients with more rounds of ${ }^{131} \mathrm{I}$ administration or surgery, which might additionally affect the change in thyroglobulin level caused by RAT, the median $\mathrm{Tg}_{\text {on }}$ level in the remaining 213 patients significantly decreased over time. Similar trends for change in median $\mathrm{Tg}_{\text {on }}$ level in patients with hyperthyroglobulinemia primarily caused by thyroid remnant, biochemical disease, and structural or functional disease were found (Fig. 6).

\section{DISCUSSION}

In this prospective multicenter study, we comprehensively reported the outcomes of RAT in a cohort of 254 TT-DTC patients with unexplained postoperative hyperthyroglobulinemia. Along with the non-S/FIR rate of $81.5 \%$, a cause of biochemical, functional, or structural disease and an intermediate to high risk were deemed present in $82.7 \%$ and $94.1 \%$ of all eligible subjects, respectively, which was reached by a sufficient follow-up after RAT with an initial administration of $5.55 \mathrm{GBq}$ of ${ }^{131} \mathrm{I}$. These novel findings may play a vital role in elucidating the indication for RAT, facilitating nuclear medicine practice and interdisciplinary communications.

Instead of the latest version, the original risk stratification protocol derived from the 2009 ATA guidelines was adopted and feasibly applied in this study, avoiding commonly insufficient detailed data on lymph node involvement and molecular features, which have been additionally suggested by the 2015 ATA guidelines. In addition, the risks were merely assessed before and soon after RAT, evading potential replacement by response categorization-the so-called dynamic risk stratification in some previous studies $(13,14)$. As a result, up to $93.3 \%$ of TT-DTC patients with unexplained postoperative hyperthyroglobulinemia were initially classified as being at intermediate to high risk by the ATA guidelines during the interval between surgery and RAT. Unexpectedly, the distributions of risk before and after RAT were similar,

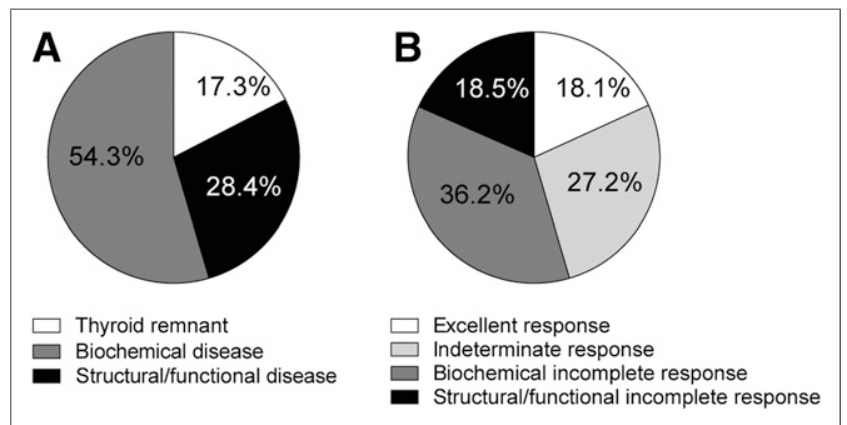

FIGURE 5. Causal distribution of unexplained postoperative hyperthyroglobulinemia after RAT (A) and therapeutic response to RAT (B) $(n=254)$. 
TABLE 2

Causal Classification Regarding Postoperative Risk of prmDTC in TT-DTC Patients with Unexplained Hyperthyroglobulinemia $(n=254)$

\begin{tabular}{lcccc}
\hline \multicolumn{1}{c}{ Risk } & Total & Thyroid remnant & Biochemical disease & Structural or functional disease \\
\hline Low & $17(6.7)$ & $5(29.4)$ & $8(47.1)$ & $4(23.5)$ \\
Intermediate & $225(88.6)$ & $36(16.0)$ & $123(54.7)$ & $66(29.3)$ \\
High & $12(4.7)$ & $3(25.0)$ & $7(58.3)$ & $2(16.7)$
\end{tabular}

Data are number followed by percentage in parentheses.

indicating a stable and predominantly intermediate- to high-risk stratification in this commonly encountered entity. This finding may be interpreted in the light of the strictly controlled indications for total thyroidectomy, the rigorous exclusion criteria of this study, and a pathologic feature-predominant risk stratification scheme. Notably, although fewer than $1 \%$ of patients initially ranked as at intermediate risk were reclassified as being at high risk immediately after RAT, as many as $11.8 \%$ of patients initially classified as at low risk were reclassified as being at intermediate risk via post-RAT scanning. This modulation suggests that initially low-risk TT-DTC patients with hyperthyroglobulinemia may need more active monitoring and aggressive intervention instead of observation or RRA-a possibility that necessitates randomized controlled studies to evaluate the impact of RAT on prognosis (15).

During a median follow-up of $10.6 \mathrm{mo}$, hyperthyroglobulinemia was attributed to 3 well-defined causes. When $5.55 \mathrm{GBq}$ of ${ }^{131} \mathrm{I}$ were used, hyperthyroglobulinemia was finally attributed to a thyroid remnant in only $17.3 \%$ of patients. Considering the high efficacy of ${ }^{131} \mathrm{I}$ in RRA as recently demonstrated by our team (11), we believe that such a dose is more competent for smaller thyroid remnants in the current study. Meanwhile, more than half the patients with unexplained hyperthyroglobulinemia were causally categorized as having biochemical disease, indicating the existence of subclinical lesions, which are most suitable for RAT and regular follow-up under TSH suppression (16). Although a significant difference in median $\mathrm{Tg}_{\text {off }}$ level was found among the 3 causal groups, it is currently difficult to use thyroglobulin level to efficiently separate patients with structural or functional disease from those with a thyroid remnant or biochemical disease before RAT, much less to use radioiodine uptake. In addition, distant metastases were identified by post-RAT scanning in fewer than
$1 \%$ of patients with no apparent anatomic abnormalities, but a favorable status after active treatment was noted. We tentatively conclude that the total tumor burden might be relatively light and that RAT with $5.55 \mathrm{GBq}$ of ${ }^{131} \mathrm{I}$ can identify and treat the lesions competently.

It has been acknowledged that patients with S/FIR require a more intensive follow-up than those with non-S/FIR $(1,17,18)$. Conversely, a non-S/FIR means a less than $1 \%$ rate of diseasespecific death $(1,19)$. The current study revealed no significant difference in response to RAT among the 3 risk groups, demonstrating that RAT using a high activity of $5.55 \mathrm{GBq}$ can yield similar short-term efficacies among the 3-tiered risk groups.

In addition to acting as an efficient biomarker for postoperative disease status, thyroglobulin has long been recognized as a reliable therapeutic response evaluation tool $(15,20,21)$. In our study, a significant decline in thyroglobulin level during as long as $1 \mathrm{y}$ of follow-up in RAT-treated patients was found regardless of the cause of hyperthyroglobulinemia, as may be explained by both the sustained destroying effect of ${ }^{131} \mathrm{I}$ on the thyroid remnant or invisible ${ }^{131} \mathrm{I}$-avid prmDTC lesions and the continuously sufficient suppression of TSH $(18,22)$. Since it has been reported that patients who had negative findings on posttherapeutic ${ }^{131} \mathrm{I}$ scanning, ultrasonography, CT, and ${ }^{18}$ F-FDG PET/CT but elevated thyroglobulin rarely showed positive findings on the second ${ }^{131}$ I scan (23), we recommended that patients without visualized ${ }^{131} \mathrm{I}$-avid lesions be monitored under continued TSH suppression in the first year after RAT. Moreover, since the thyroglobulin level just before RAT might be influenced by both TSH level and interval between total thyroidectomy and RAT, thyroid hormone withdrawal for $4 \mathrm{wk}$ after total thyroidectomy when the median TSH level had climbed up to $100 \mathrm{mIU} / \mathrm{mL}$ (range, $49.5-100 \mathrm{mIU} / \mathrm{mL}$ ) adequately ensured both cleanup of existing serum thyroglobulin

TABLE 3

Comparisons of RAIU, TSH, Tg off, and TgAb Levels Among 3 Primary Causes of Unexplained Hyperthyroglobulinemia in TT-DTC Patients $(n=254)$

\begin{tabular}{lcccc}
\hline \multicolumn{1}{c}{ Factor } & Thyroid remnant & Biochemical disease & Structural or functional disease & $P$ \\
\hline RAIU $(\%)$ & $5.2 \pm 3.2$ & $4.6 \pm 3.1$ & $4.9 \pm 3.3$ & 0.964 \\
\hline $\mathrm{TSH}(\mathrm{mlU} / \mathrm{L})$ & $100(49.5-100)$ & $100(49.6-100)$ & $100(56.6-100)$ & 0.225 \\
$\mathrm{Tg}_{\text {off }}(\mathrm{ng} / \mathrm{mL})$ & $17.0(12.7-25.5)$ & $26.9(16.6-48.9)$ & $33.5(22.2-67.8)$ & 0.000 \\
$\mathrm{TgAb}(\mathrm{IU} / \mathrm{mL})$ & $10(10-64.4)$ & $10(10-80.34)$ & $10.5(10-62.9)$ & 0.227
\end{tabular}

RAIU = radioactive iodine uptake; IQR $=$ interquartile range.

Data are mean \pm SD or median followed by range in parentheses. 

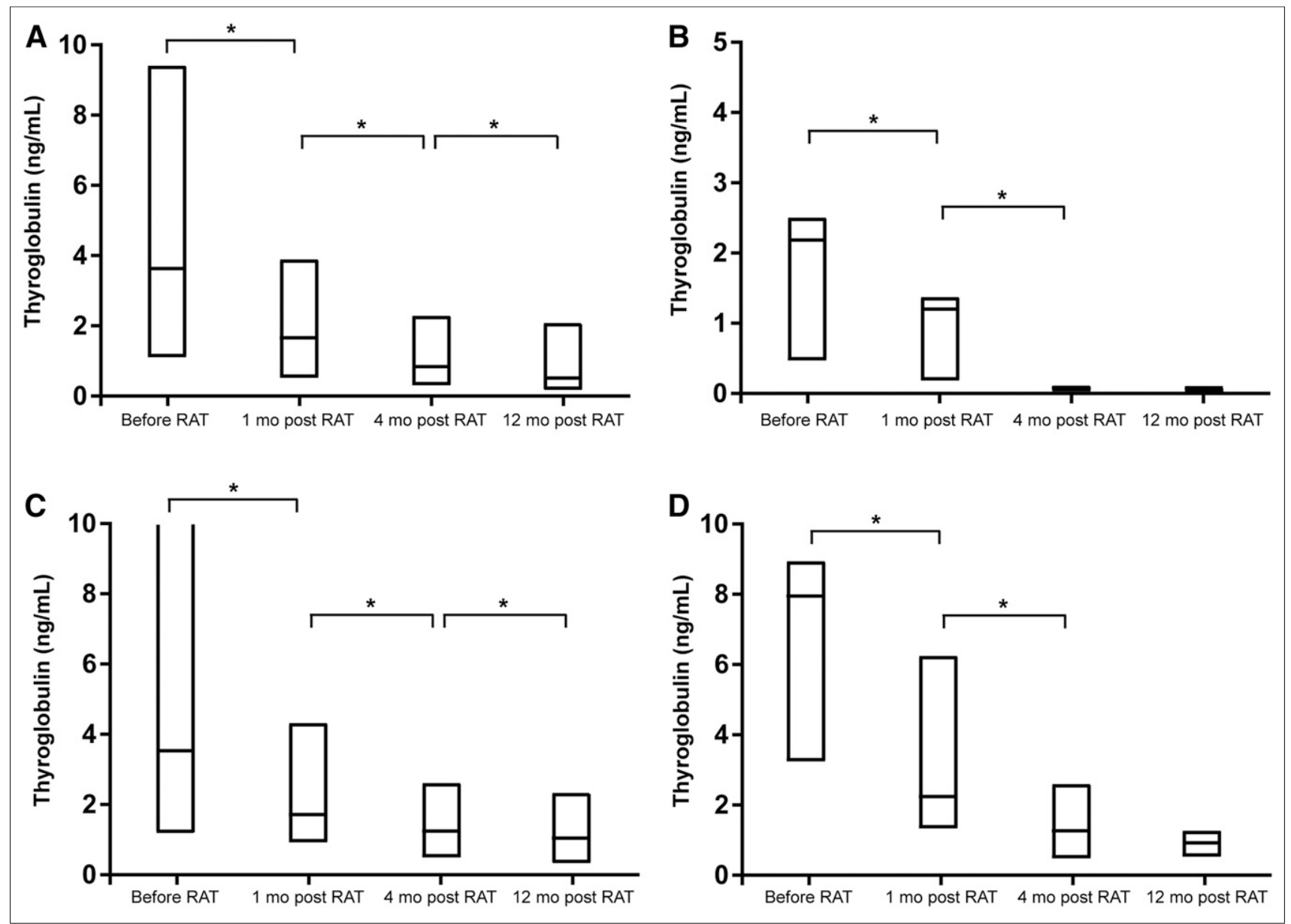

FIGURE 6. Change in suppressed thyroglobulin level in patients with unexplained hyperthyroglobulinemia who received only RAT. (A) All patients $(n=213)$. (B, C, and D) Patients with unexplained hyperthyroglobulinemia causally attributed to thyroid remnant $(n=44)$, biochemical disease $(n=138)$, and structural or functional disease $(n=31)$, respectively. Data are expressed as median with interquartile range. ${ }^{\star} P<0.05$.

immediately after total thyroidectomy and exposure of thyroglobulin deriving from a microscopic thyroid remnant or prmDTC lesion (1).

Although the term RAT has been raised for decades and attempts were recently made to define it as " ${ }^{131} \mathrm{I}$ administered to destroy subclinical tumor deposits after surgical resection of all known primary tumor tissue and metastatic foci," a clear definition of "subclinical tumor deposits" was not achieved by the joint statement (24). Even more confusingly, the statement has also mentioned that patients with biochemical evidence of disease can also be candidates for RT, as are patients with structural or functional disease $(1,24)$. The above difficulties and inconsistencies may be caused by the severe overlap in thyroglobulin level and potentially simultaneous goals among the 3 clinical scenarios (RRA, RAT, and RT) and the absence of precisely differential techniques, necessitating diagnostic radioiodine scanning and other imaging modalities (25). As was demonstrated by the current study, unexplained hyperthyroglobulinemia in TT-DTC patients represents an apparent indicator for RAT irrespective of the initial risk stratification, since over $90 \%$ of patients with this entity were stratified as being at intermediate to high risk and $82.7 \%$ of patients were finally attributed to have biochemical, functional, or structural disease. Therefore, we believe that our findings may ameliorate the relatively vague indication of RAT compared with those of RRA and RT and constructively promote scientific dialogue.

The study had some limitations. First, because of long-term controversies, diagnostic radioiodine imaging, which might have explained the cause of hyperthyroglobulinemia in part before RAT, was not incorporated in our study. Second, since the optimal administered activity of ${ }^{131} \mathrm{I}$ in RAT had not been determined, our patients received a fixed activity of ${ }^{131} \mathrm{I}$; thus, studies comparing the outcomes and side effects of multiple doses are still needed. Third, a forthcoming randomized control study assessing longterm outcomes may strengthen the significance of RAT by compensating this single-arm study.

\section{CONCLUSION}

Our prospective multicenter study demonstrated that unexplained hyperthyroglobulinemia in TT-DTC patients represents an appropriate indicator for RAT, since there was found to be an intermediate to high risk of carrying prmDTC in greater than $90 \%$ of patients, and RAT with an activity of $5.55 \mathrm{GBq}$ revealed biochemical, functional, or structural disease and yielded non-S/ FIR in more than $80 \%$ of patients. 


\section{DISCLOSURE}

This work was supported by the National Natural Science Foundation of China (grant 81671711). No other potential conflict of interest relevant to this article was reported.

KEY POINTS

QUESTION: How should TT-DTC patients with unexplained hyperthyroglobulinemia be treated?

PERTINENT FINDINGS: Our prospective multicenter cohort study demonstrated that over $90 \%$ of TT-DTC patients with unexplained hyperthyroglobulinemia were stratified as being at intermediate to high risk, and RAT at a dose of $5.55 \mathrm{GBq}$ revealed biochemical, functional, or structural disease and yielded a nonstructurally or functionally incomplete response in more than $80 \%$ of subjects. Distribution for either cause of hyperthyroglobulinemia or response to RAT was comparable among the 3 postoperative risk groups.

IMPLICATIONS FOR PATIENT CARE: These findings recommend TT-DTC patients with unexplained hyperthyroglobulinemia as candidates for RAT irrespective of postoperative risk stratification.

\section{REFERENCE}

1. Haugen BR, Alexander EK, Bible KC, et al. 2015 American Thyroid Association management guidelines for adult patients with thyroid nodules and differentiated thyroid cancer: the American Thyroid Association guidelines task force on thyroid nodules and differentiated thyroid cancer. Thyroid. 2016;26:1-133.

2. Choi Y, Jung SL. Efficacy and safety of thermal ablation techniques for the treatment of primary papillary thyroid microcarcinoma: a systematic review and meta-analysis. Thyroid. 2020;30:720-731.

3. Molinaro E, Campopiano MC, Pieruzzi L, et al. Active surveillance in papillary thyroid microcarcinomas is feasible and safe: experience at a single Italian center. J Clin Endocrinol Metab. 2020;105:dgz113.

4. Pelttari H, Valimaki MJ, Loyttyniemi E, Schalin-Jantti C. Post-ablative serum thyroglobulin is an independent predictor of recurrence in low-risk differentiated thyroid carcinoma: a 16-year follow-up study. Eur J Endocrinol. 2010;163:757-763.

5. Giovanella L, Ceriani L, Ghelfo A, Keller F. Thyroglobulin assay 4 weeks after thyroidectomy predicts outcome in low-risk papillary thyroid carcinoma. Clin Chem Lab Med. 2005;43:843-847.

6. Webb RC, Howard RS, Stojadinovic A, et al. The utility of serum thyroglobulin measurement at the time of remnant ablation for predicting disease-free status in patients with differentiated thyroid cancer: a meta-analysis involving 3947 patients. J Clin Endocrinol Metab. 2012;97:2754-2763.

7. Piccardo A, Arecco F, Puntoni M, et al. Focus on high-risk DTC patients high postoperative serum thyroglobulin: high postoperative serum thyroglobulin level is a strong predictor of disease persistence and is associated to progression-free survival and overall survival. Clin Nucl Med. 2013;38:18-24.

8. Kim HK, Yoon JH, Cho JS, Kwon SY, Yoo SW, Kang HC. The clinical meaning of pre- and post-ablation thyroglobulin levels at first radioiodine therapy in patients with papillary thyroid cancer. Korean J Intern Med. July 29, 2019 [Epub ahead of print].
9. Cooper DS, Doherty GM, Haugen BR, et al. Revised American Thyroid Association management guidelines for patients with thyroid nodules and differentiated thyroid cancer. Thyroid. 2009;19:1167-1214.

10. Yang X, Liang J, Li T, Zhao T, Lin Y. Preablative stimulated thyroglobulin correlates to new therapy response system in differentiated thyroid cancer. J Clin Endocrinol Metab. 2016;101:1307-1313.

11. Jin Y, Ruan M, Cheng L, et al. Radioiodine uptake and thyroglobulin-guided radioiodine remnant ablation in patients with differentiated thyroid cancer: a prospective, randomized, open-label, controlled trial. Thyroid. 2019;29:101110.

12. Chen L, Luo Q, Shen Y, et al. Incremental value of ${ }^{131}$ I SPECT/CT in the management of patients with differentiated thyroid carcinoma. J Nucl Med. 2008;49:1952-1957.

13. Krajewska J, Chmielik E, Jarzab B. Dynamic risk stratification in the follow-up of thyroid cancer: what is still to be discovered in 2017? Endocr Relat Cancer. 2017;24:R387-R402.

14. Castagna MG, Maino F, Cipri C, et al. Delayed risk stratification, to include the response to initial treatment (surgery and radioiodine ablation), has better outcome predictivity in differentiated thyroid cancer patients. Eur J Endocrinol. 2011;165:441-446.

15. Kim TY, Kim WB, Kim ES, et al. Serum thyroglobulin levels at the time of ${ }^{131} \mathrm{I}$ remnant ablation just after thyroidectomy are useful for early prediction of clinical recurrence in low-risk patients with differentiated thyroid carcinoma. J Clin Endocrinol Metab. 2005;90:1440-1445.

16. Pitoia F, Bueno F, Urciuoli C, Abelleira E, Cross G, Tuttle RM. Outcomes of patients with differentiated thyroid cancer risk-stratified according to the American Thyroid Association and Latin American Thyroid Society risk of recurrence classification systems. Thyroid. 2013;23:1401-1407.

17. Tuttle RM, Tala H, Shah J, et al. Estimating risk of recurrence in differentiated thyroid cancer after total thyroidectomy and radioactive iodine remnant ablation: using response to therapy variables to modify the initial risk estimates predicted by the new American Thyroid Association staging system. Thyroid. 2010;20: 1341-1349.

18. Vaisman F, Momesso D, Bulzico DA, et al. Spontaneous remission in thyroid cancer patients after biochemical incomplete response to initial therapy. Clin Endocrinol (Oxf). 2012;77:132-138.

19. Seejore K, Gerrard GE, Gill VM, Murray RD. Can we discharge dynamically risk-stratified low-risk (excellent response to treatment) thyroid cancer patients after 5 years of follow-up? Clin Oncol (R Coll Radiol). 2019;31:219224.

20. Zanella A, Scheffel RS, Pasa MW, Dora JM, Maia AL. Role of postoperative stimulated thyroglobulin as prognostic factor for differentiated thyroid cancer in children and adolescents. Thyroid. 2017;27:787-792.

21. Matrone A, Gambale C, Piaggi P, et al. Postoperative thyroglobulin and neck ultrasound in the risk restratification and decision to perform ${ }^{131} \mathrm{I}$ ablation. $J$ Clin Endocrinol Metab. 2017;102:893-902.

22. Biko J, Reiners C, Kreissl MC, Verburg FA, Demidchik Y, Drozd V. Favourable course of disease after incomplete remission on ${ }^{131} \mathrm{I}$ therapy in children with pulmonary metastases of papillary thyroid carcinoma: 10 years follow-up. Eur J Nucl Med Mol Imaging. 2011;38:651-655.

23. Rosario PW, Mourao GF, dos Santos JB, Calsolari MR. Is empirical radioactive iodine therapy still a valid approach to patients with thyroid cancer and elevated thyroglobulin? Thyroid. 2014;24:533-536.

24. Tuttle RM, Ahuja S, Avram AM, et al. Controversies, consensus, and collaboration in the use of ${ }^{131} \mathrm{I}$ therapy in differentiated thyroid cancer: a joint statement from the American Thyroid Association, the European Association of Nuclear Medicine, the Society of Nuclear Medicine and Molecular Imaging, and the European Thyroid Association. Thyroid. 2019;29:461-470.

25. Avram AM, Esfandiari NH, Wong KK. Preablation 131-I scans with SPECT/CT contribute to thyroid cancer risk stratification and 131-I therapy planning. J Clin Endocrinol Metab. 2015;100:1895-1902. 